\title{
Editorial
}

\section{The Human Document Project and Challenges}

\author{
Andreas Manz \\ Founding Editor-in-Chief, Korea Institute of Science and Technolgy (KIST), KIST Europe \\ Forschungsgesellschaft mbH, Universität des Saarlandes, Campus E 71, D-66123 Saarbruecken, \\ Germany; E-Mail: manz@kist-europe.de; Tel. +49-681-9382-210; Fax: +49-681-9382-109.
}

Received: 19 July 2010 / Accepted: 19 July 2010 / Published: 20 July 2010

Literature, newspapers or science use the internet, paper and written language for documenting their contents and transmitting it to the readers. The time scale for this is typically a human generation or can be much less. Technically speaking, printed paper, as such, will not necessarily survive very much longer. The computerized modern world has boosted the storage and accessibility of much more information. However, this has not improved the survival time scale [1].

Heart beat frequency, body size of an organism and time scales of reaction may correlate. We have developed a civilization which overcame these biological hurdles through, e.g., medicine and technology for self-protection. However, human thinking is mostly limited to short timescales, in the best case to one or two generations ahead. Long-term documentation has only occurred related to religion and the idea of "eternal life", or purely by accident. Ancient cultures documented themselves on cave paintings, petroglyphs and rock carvings. Clay plates and large architectural objects have demonstrated lifetimes of thousands of years. At least, that is how it appears to be today. We simply have no evidence of other forms of communication, since most has disappeared with time. We can find that Homo erectus or Homo neandertalensis was able to prepare fire, because this is documented in inorganic traces. However, perhaps there was a scientific understanding or expression of art that may have vanished over the years. How can we know?

What will remain of today's efforts of the arts and sciences, not to speak about the many aspects of everyday life, in say 1,000,000 years?

It may be comparable to the remains we currently have of Homo erectus and his lifestyle. Computers will have corroded (except a few silicon chips), paper all gone, houses disappeared and with it most other items we use in everyday life. Of course, we can hope for a community which passes down information as the medieval monasteries did in copying Aristotle's books. However, we have no guarantee that there will be a smooth transition or continuous development in Homo sapiens, as we can observe in the last one million years. Historians speak of hundreds of years, archaeologists of 
hundreds of thousands of years and astronomers or geologists handle the real time scales, which we currently believe play no role for Homo sapiens. That might be wrong. In a similar way, as there was centuries of debate about the geocentric vs. heliocentric viewpoint for astronomy, there could be a debate about relevant timescales. We may not have a (very geological) prehistory of much more than one million years, but we may have a future of intelligent life on this planet of much longer! I am not saying that it will be Homo sapiens; it might as well be another humanoid species, another mammal or even social insects. Speculation of that kind is to be avoided, but I am sure you get my point...

\section{References and Notes}

1. The Human Document Project website: http://www.humandocument.org/.

(C) 2010 by the authors; licensee MDPI, Basel, Switzerland. This article is an Open Access article distributed under the terms and conditions of the Creative Commons Attribution license (http://creativecommons.org/licenses/by/3.0/). 